04

\title{
Электрофизические и тепловые параметры плазменной струи атмосферного давления в гелии при возбуждении синусоидальным и импульсным напряжением
}

\author{
(C) П.П. Гугин ${ }^{1}$, Д.Э. Закревский ${ }^{1,2, \uparrow}$, Е.В. Милахина ${ }^{1,2}$ \\ ${ }^{1}$ Институт физики полупроводников им. А.В. Ржанова СО РАН, Новосибирск, Россия \\ ${ }^{2}$ Новосибирский государственный технический университет, Новосибирск, Россия \\ ๑ E-mail: zakrdm@isp.nsc.ru
}

Поступило в Редакцию 27 июля 2021 г.

В окончательной редакции 10 августа 2021 г.

Принято к публикации 11 августа 2021г.

Проведены сравнительные исследования генерации холодной плазменной струи атмосферного давления в гелии при возбуждении синусоидальным напряжением и однополярными положительными импульсами при напряжении $2-6 \mathrm{kV}$ для медицинских приложений. Показано, что достигаемые параметры идентичны, но отсутствие эффекта частотной самоорганизации стримерного пробоя, приводящего к нерегулярности в распространении стримеров при импульсном возбуждении, делает его предпочтительным в биофизических экспериментах. Ограничивающим фактором воздействия плазменной струи является достигаемая температура мишени, которая может быть снижена ограничением длительности тока.

Ключевые слова: плазменная струя атмосферного давления, возбуждение синусоидальным и импульсным напряжением, температура.

DOI: 10.21883/PJTF.2021.22.51726.18977

Внимание к исследованиям генерации плазмы атмосферного давления, в частности холодного разряда, который при определенных условиях выходит за пределы разрядной зоны, по принятой в последнее время терминологии - холодной плазменной струи (ХПС), связано с ее применением в качестве инструмента в плазменной медицине (см., например, [1-5]).

Воздействие плазменных образований на биологические объекты стимулирует химические реакции в газовой фазе и жидкости на границе плазма-биологический объект [2-4,6]. Неравновесный характер плазмы позволяет создавать химически активные вещества без чрезмерного нагрева газа и воздействовать на мишени, в том числе живые организмы, чувствительные к нагреву. В настоящее время воздействие ХПС на биологические объекты является одним из подходов, обеспечивающих активацию процессов подавления жизнеспособности раковых клеток различного гистологического происхождения [7].

Во многих биофизических экспериментах используется источник ХПС, генерируемой при приложении к электродам синусоидального напряжения. Стримеры, инициируемые на каждой положительной волне напряжения, распространяются в потоке инертного газа в окружающее пространство и формируют плазменную струю. В работе [8] при воздействии ХПС на диэлектрическую мишень обнаружен эффект частотной самоорганизации стримерного пробоя. Частота импульсов тока, регистрируемых вблизи мишени $\left(f_{I}\right)$, зависит от амплитуды $U$, частоты $f_{0}$ прикладываемого синусоидального напряжения, расстояния $z$ от сопла до мишени и ее типа и не совпадает с $f_{0}$. Этот эффект зависит от условий в промежутке сопло-мишень и определяется соотношением плотности плазмы, образующейся в головке стримера, и остаточной плотности плазмы над поверхностью мишени. Все электромагнитные и химические процессы вблизи мишени зависят от частоты контактов плазмы с мишенью. Нерегулируемый процесс изменения параметров ХПС приводит к неконтролируемым и часто невоспроизводимым условиям воздействия на мишень, способен вызвать локальный перегрев живых тканей из-за повышения температуры плазмообразующего газа и привести к дестабилизации клеточных белков. Для применения ХПС в лабораторных исследованиях in vitro и in vivo необходимо контролировать ее параметры, чтобы обеспечить эффективность и безопасность воздействия на живые ткани.

Целью настоящей работы является сравнительное исследование параметров воздействия ХПС на мишень (ток, частотные зависимости, температура мишени, спектральный состав струи над поверхностью мишени) при возбуждении синусоидальным напряжением и однополярными положительными импульсами применительно к биофизическим исследованиям.

Для генерации ХПС использовались генератор синусоидального напряжения на фиксированных частотах $f_{0} \approx 13,21,52 \mathrm{kHz}$, генератор однополярных положительных импульсов с регулируемой частотой следования $f=3-30 \mathrm{kHz}$. В обоих случаях амплитуда напряжения $U$ лежала в диапазоне $2-6 \mathrm{kV}$ и ограничивалась для обеспечения безопасных условий воздействия ХПС на живые объекты. 

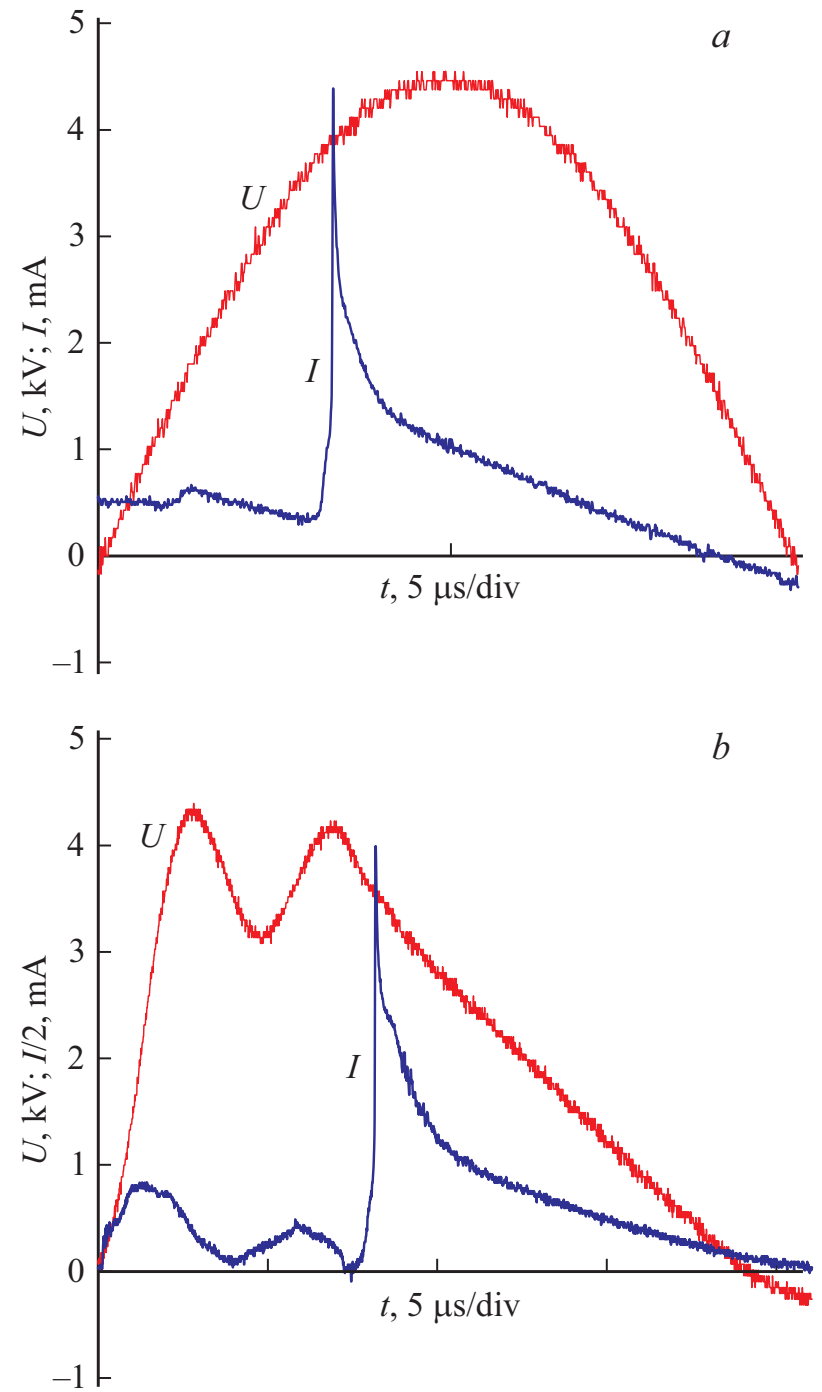

Рис. 1. Осциллограммы напряжения на высоковольтном электроде $U$ и тока на коллекторе $I$ при возбуждении синусоидальным $(a)$ и импульсным $(b)$ напряжением. $f_{0} \approx f \approx 13 \mathrm{kHz}$.

Источник ХПС представлял собой коаксиальный диэлектрический канал длиной $100 \mathrm{~mm}$ с внутренним диаметром $8 \mathrm{~mm}$ с соплом на конце канала в виде капилляра диаметром $2.3 \mathrm{~mm}$ [8-10]. Разрядная зона образована двумя электродами. К внутреннему стержневому электроду, расположенному в центре канала длиной $50 \mathrm{~mm}$ и диаметром $2 \mathrm{~mm}$, относительно кольцевого заземленного электрода, размещенного снаружи канала, прикладывалось возбуждающее напряжение. Измерения напряжения $U$ проводились с высокоомным делителем. На расстоянии от сопла $z=20 \mathrm{~mm}$ перпендикулярно оси распространения ХПС размещался металлический электрод-коллектор, представляющий собой медную подложку. Величина $z$ выбиралась исходя из типичных условий экспериментов по воздействию ХПС на культуральные среды и подопытных животных. Заземление коллектора через малоиндуктивное сопротивление поз- воляло регистрировать импульс тока $I$, попадающего на подложку. Ее использование обосновано и тем фактом, что в промежутке плазменный стример-заземленный электрод реализуется конфигурация электрического поля с повышенной напряженностью. Это совместно с увеличением амплитуды и частоты возбуждающего напряжения приводит к интенсификации генерации активных радикалов в зоне контакта ХПС с мишенью. Для этого в биологических экспериментах под планшетом с объектами специально размещается вспомогательный заземленный электрод $[4,9,10]$.

При прокачке через канал рабочего газа - гелия марки А (чистота 99.995\%) - со скоростью потока $v>11 /$ min и при приложении к электродам синусоидального напряжения с $U \approx 0.7 \mathrm{kV}$ или однополярных положительных импульсов с $U \approx 1.1 \mathrm{kV}$ в разрядном промежутке зажигался разряд и при превышении соответственно $U>1 \mathrm{kV}$ и $U>1.5 \mathrm{kV}$ формировалась плазменная струя, выходящая за пределы канала. Локализация в пространстве плазменного образования зависит от геометрии канала, размера капилляра, скорости потока и напряжения. В оптимальных условиях при $U=3-5 \mathrm{kV}$, $v=5-101 / \mathrm{min}$ длина струи достигала $70 \mathrm{~mm}$.

Осциллограммы напряжения и тока коллектора для обоих случаев возбуждения при $U \approx 4.5 \mathrm{kV}$, $f_{0} \approx f \approx 13 \mathrm{kHz}$ представлены на рис. $1, a, b$. Эксперименты показывают, что ток на мишени имеет сложный характер и состоит из быстрой части (непосредственно стримера длительностью до $100 \mathrm{~ns}$ ) и медленной части (длительностью 5-10 $\mu \mathrm{s}$ ).

На рис. 2, a представлены зависимости амплитуды тока коллектора от амплитуды прикладываемого напряжения $I(U)$ для различных скоростей потока газа при синусоидальном и импульсном возбуждении. Видно, что зависимости однотипны: при постоянной скорости потока $I$ растет с увеличением $U$ с последующим насыщением. Максимально достигаемые амплитуды тока в обоих случаях были сравнимы и не превышали $\sim 20 \mathrm{~mA}$. При постоянной частоте с увеличением $U$ время задержки развития разряда $\tau_{d}$ (время между началом положительной части напряжения и появлением тока на коллекторе) уменьшается (рис. $2, b$ ).

Исследования параметров ХПС при возбуждении синусоидальным напряжением с $f_{0} \approx 52 \mathrm{kHz}$, как и в [8], показали, что частота касания мишени импульсами тока $f_{I}$ в зависимости от условий в промежутке сопло-мишень равна или кратна частоте прикладываемого напряжения: $f_{I} \approx f_{0}, f_{0} / 2, f_{0} / 3$ или $f_{0} / 4$. Минимальная частота составила $f_{I} \approx 13 \mathrm{kHz}$. В отличие от случая синусоидального напряжения [8] при импульсном возбуждении обнаружено, что $f_{I}=f_{0}$. Стабильное функционирование ХПС сохранялось при всех исследованных параметрах $U, v, z$ и типах мишени. Это позволило измерить частотную характеристику $I(f)$. При $U=5 \mathrm{kV}$ и $v=4.5 \mathrm{l} / \mathrm{min}$ амплитуда регистрируемого тока экспоненциально уменьшается при повышении частоты следования импульсов $f$ (рис. $2, c)$. Исследования 
спектроскопических параметров струи над поверхностью культуральной среды при прочих равных условиях при обоих типах возбуждения не выявили существенных различий в составе и интенсивностях генерируемых активных компонентов.

Ограничивающим фактором воздействия ХПС на биологические объекты при увеличении ее мощности является нагрев поверхности мишени в зоне контакта с плазмой. Эксперименты показали, что температура $T$ диэлектрической подложки (керамика $\mathrm{Al}_{2} \mathrm{O}_{3}$ толщиной $1 \mathrm{~mm}$ ) на заземленном электроде при воздействии на нее ХПС устанавливалась за $\sim 1 \mathrm{~min}$. При возбуждении ХПС синусоидальным напряжением с $U=4 \mathrm{kV}$, $v \approx 91 / \mathrm{min}$ при $f_{0}=13 \mathrm{kHz}$ температура подложки достигала $T \approx 34^{\circ} \mathrm{C}$, при $f_{0} \approx 21 \mathrm{kHz}-T \approx 50^{\circ} \mathrm{C}$, при
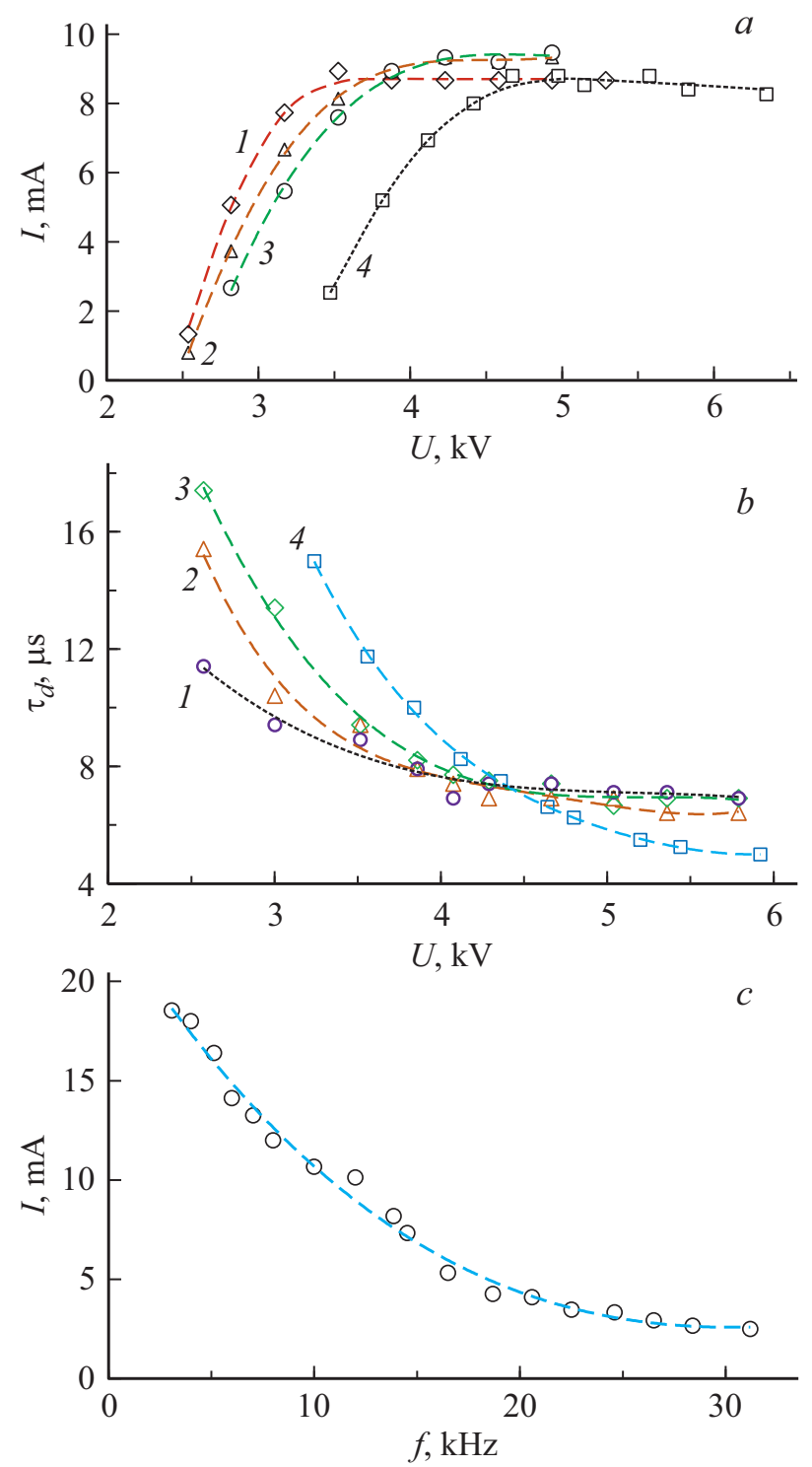

Рис. 2. Зависимости $I(U)(a)$ и $\tau_{d}(U)(b) . \quad v=3$ (1), 4.5 $(2,4), 61 / \min (3) ; f_{0} / 4 \approx f \approx 13 \mathrm{kHz}$; синусоидальное $(1-3)$ и импульсное (4) возбуждение. $c-I(f), U=5 \mathrm{kV}, v=4.5 \mathrm{l} / \mathrm{min}$, импульсное возбуждение.

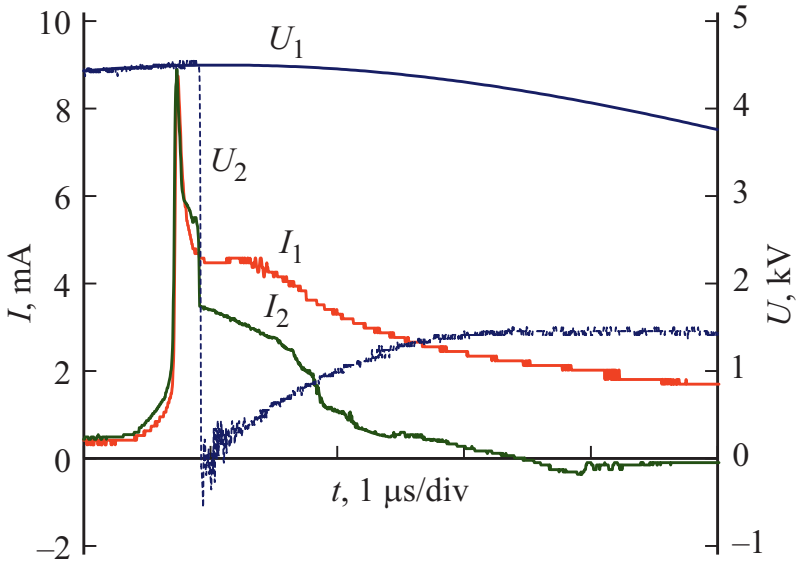

Рис. 3. Осциллограммы напряжения на высоковольтном электроде и тока на коллекторе в случаях без ограничения $\left(U_{1}, I_{1}\right)$ и с ограничением $\left(U_{2}, I_{2}\right)$ длительности импульса тока. $f_{0}=21 \mathrm{kHz}, v \approx 91 / \mathrm{min}$.

$f_{0} \approx 52 \mathrm{kHz}-T \approx 87^{\circ} \mathrm{C}$. Очевидно, что достигаемые температуры мишени опасны для живых биологических объектов. Уменьшить нагрев можно, ограничивая длительность импульса тока. Для этого в работе использовался ключ - тиратрон ТГИ1-270/12, анод которого подключался к потенциальному электроду источника ХПС. Момент срабатывания ключа определялся сигналом с оптического датчика, регистрирующего интегральный сигнал свечения плазмы при прохождении тока ХПС, что в совокупности с управляемой задержкой позволяло контролируемо ограничивать импульс возбуждающего напряжения.

На рис. 3 представлены совмещенные осциллограммы напряжения и тока при синусоидальном возбуждении с $U=4.5 \mathrm{kV}, f_{0}=21 \mathrm{kHz}$ и $v \approx 91 / \mathrm{min}$ в случаях без ограничения и с ограничением длительности тока. В последнем случае температура мишени уменьшалась на $\sim 20 \%$ и с увеличением $U$ эта разница нарастала. Аналогичные результаты получены и при возбуждении импульсным напряжением.

Таким образом, показано, что достигаемые параметры воздействия ХПС на мишень при синусоидальном и импульсном возбуждении идентичны при прочих равных условиях, но отсутствие эффекта частотной самоорганизации стримерного пробоя при импульсном возбуждении делает последнее более предпочтительным в биофизических экспериментах. Общим ограничивающим фактором в обоих типах возбуждения ХПС является температура мишени, которая может превышать $42^{\circ} \mathrm{C}$ и быть опасной для живых объектов; еe снижение возможно за счет ограничения длительности тока ХПС.

\section{Финансирование работы}

Исследование выполнено при финансовой поддержке Российского фонда фундаментальных исследований и 
Правительства Новосибирской области в рамках научного проекта № 20-48-540019 и гранта Российского научного фонда № 19-19-00255.

\section{Соблюдение этических стандартов}

Настоящая статья не содержит каких-либо исследований с использованием животных в качестве объектов.

\section{Конфликт интересов}

Авторы заявляют, что у них нет конфликта интересов.

\section{Список литературы}

[1] Plasma technology for biomedical applications, ed by E. Martines (MDPI, Basel, 2020). DOI: 10.3390/books978-3-03928-737-6

[2] J.C. Harley, N. Suchowerska, D.R. McKenzie, Biophys. Rev., 12, 989 (2020). DOI: 10.1007/s12551-020-00743-Z

[3] A. Malyavko, D. Yan, Q. Wang, A.L. Klein, K.C. Patel, J.H. Shermand, M. Keidar, Mater. Adv., 1, 1494 (2020). DOI: 0.1039/d0ma00329h

[4] L. Lin, M. Keidar, Appl. Phys. Rev., 8, 011306 (2021). DOI: $10.1063 / 5.0022534$

[5] М.Ю. Герасименко, Т.Н. Зайцева, И.С. Евстигнеева, Физическая и реабилитационная медицина, Медицинская реабилитация, 1 (3), 79 (2019).

DOI: $10.36425 / 2658-6843-2019-3-79-89$

[6] X. Lu, G.V. Naidis, M. Laroussi, S. Reuter, D.B. Graves, K. Ostrikov, Phys. Rep., 630, 1 (2016). DOI: 10.1016/j.physrep.2016.03.003

[7] M. Keidar, D. Yan, J.H. Sherman, Cold plasma cancer therapy (Morgan \& Claypool Publ., 2019).

[8] I.V. Schweigert, A.L. Alexandrov, D.E. Zakrevsky, Plasma Sources Sci. Technol., 29, 12LT02 (2020). DOI: 10.1088/1361-6595/abc93f

[9] I. Schweigert, Dm. Zakrevsky, P. Gugin, E. Yelak, E. Golubitskaya, O. Troitskaya, O. Koval, Appl. Sci., 9, 4528 (2019). DOI: 10.3390/app9214528

[10] O. Troitskaya, E. Golubitskaya, M. Biryukov, M. Varlamov, P. Gugin, E. Milakhina, V. Richter, I. Schweigert, Dm. Zakrevsky, O. Koval, Int. J. Mol. Sci., 21, 2158 (2020). DOI: $10.3390 /$ ijms 21145128 